\title{
Optimal Initial Intravenous Diuretic Dose for Acute Decompensated Heart Failure: Insights from the REALITY-AHF
}

Kenji Yoshioka

Kameda Medical Center

Daichi Maeda

Juntendo University

Takahiro Okumura

Nagoya University Graduate School of Medicine

Keisuke Kida

St. Marianna University School of Medicine

Shogo Oishi

Himeji Cardiovascular Center

Eiichi Akiyama

Yokohama City University Medical Center

Satoshi Suzuki

Fukushima Medical University

Masayoshi Yamamoto

University of Tsukuba

Akira Mizukami

Kameda Medical Center

Shunsuke Kuroda

Cleveland Clinic

Nobuyuki Kagiyama

Juntendo University

Tetsuo Yamaguchi

Toranomon Hospital

Tetsuo Sasano

Tokyo Medical and Dental University

Akihiko Matsumura

Kameda Medical Center

Takeshi Kitai

National Cerebral and Cardiovascular Center

Yuya Matsue ( $\nabla$ yuya8950@gmail.com ) 
Juntendo University

\section{Research Article}

Keywords: intravenous diuretic dose, acute decompensated heart failure, REALITY- AHF

Posted Date: September 7th, 2021

DOl: https://doi.org/10.21203/rs.3.rs-847220/v1

License: (c) (i) This work is licensed under a Creative Commons Attribution 4.0 International License. Read Full License 


\section{Abstract}

Although intravenous diuretics is a cornerstone of acute heart failure treatment (AHF), its optimal initial dose is unclear. This is a post-hoc analysis of the REALITY-AHF, a prospective multicentre observational registry of AHF. The initial intravenous diuretic dose used in each patient was categorised into below, standard, or above the recommended dose groups according to guideline-recommended initial intravenous diuretic dose. The recommended dose was individualised based on the oral diuretic dose taken at admission. We compared the study endpoints, including 60-day mortality, diuretics response within six hours, and length of hospital stay (HS). Of 1,093 patients, 429, 558, and 106 were assigned to the Below, Standard, and Above groups, respectively. The diuretics response and HS were significantly greater in the Below group than in the Standard group after adjusting for covariates. Kaplan-Meier analysis indicated a significantly higher incidence of 60-day mortality in the Above group than the Standard group. This difference was retained after adjusting for other prognostic factors. Treatment with a lower than guideline-recommended intravenous diuretic dose was associated with longer HS, whereas above the guideline-recommended dose was associated with a higher 60-day mortality rate. Our results reconfirm that the guideline-recommended initial intravenous diuretic dose is feasible for AHF.

\section{Introduction}

Decongestion with intravenous (IV) diuretics is a mainstay of acute heart failure (AHF) treatment since congestion is one of the primary reasons for heart failure admission. ${ }^{1}$ Although diuretics are an effective treatment for most patients with AHF, the ideal dose of IV loop diuretics has yet to be established. The Diuretic Optimization Strategies Evaluation (DOSE) trial provided important insights regarding clinical and prognostic implications of high vs low dose loop diuretics, finding no prognostic differences. However, current guidelines recommend using the smallest amount of diuretics to provide adequate decongestion. ${ }^{2,3}$ This reflects the fact that a greater amount of loop diuretics tends to induce a stronger diuresis and greater relief of symptom. However, giving a large amount of diuretics is not always good given the adverse effects reported in patients with heart failure. ${ }^{4,5}$ Current guidelines recommend 20 to 40 mg of IV furosemide for patients with AHF not receiving oral diuretics, or an equivalent or higher dose than the oral diuretics for those already taking it. However, these recommendations have not been validated yet. ${ }^{2,3}$ Therefore, we sought to examine the current recommendations on the initial IV furosemide dose administered to patients with AHF in terms of treatment efficiency and prognostic impact, using the REALITY-AHF (Registry Focused on Very Early Presentation and Treatment in Emergency Department of Acute Heart Failure) cohort. ${ }^{6}$

\section{Results}

Of the 1,682 patients enrolled in the REALITY-AHF, 1,109 remained after excluding those not treated with furosemide within six hours of admission or treated with continuous furosemide infusion. We further excluded 26 patients with missing data on the amount of the first IV furosemide bolus. Consequently, 
1,093 patients were analysed (Supplemental Figure S1). These patients were assigned to one of three groups according to the guideline-recommended dose: Below $(n=429)$, Standard $(n=558)$, and Above ( $n$ $=106$ ). Baseline characteristics of the three groups are shown in Table 1. Significant between group differences were observed in blood pressure, presence of orthopnoea and pulmonary oedema, history of heart failure, being treated with loop diuretics, beta-blockers, and/or aldosterone blocker before admission, and levels of haemoglobin, creatinine, and blood urea nitrogen. Of note, the Below group, but not the Above group, showed patient characteristics associated with poor prognosis such as lower systolic blood pressure, less orthopnoea and pulmonary oedema, more patients with a history of heart failure, being treated with high-dose loop diuretics before admission, and poor renal function in comparison to the Standard group. 


\section{Table 1}

Baseline characteristics of the study participants

\begin{tabular}{|c|c|c|c|c|}
\hline Variables & Below & Standard & Above & $\begin{array}{l}P \\
\text { value }\end{array}$ \\
\hline$N=429$ & $N=558$ & $N=106$ & & \\
\hline Age (years) & $78(12)$ & $78(11)$ & $79(12)$ & 0.522 \\
\hline Male sex (\%) & $246(57.3)$ & $303(54.3)$ & $56(52.8)$ & 0.546 \\
\hline Systolic blood pressure (mmHg) & $146(35)$ & $158(34)$ & $157(38)$ & $<0.001$ \\
\hline Diastolic blood pressure $(\mathrm{mmHg})$ & $83(24)$ & $88(26)$ & $87(29)$ & 0.001 \\
\hline Heart rate $(\mathrm{bpm})$ & $96(26)$ & $102(30)$ & $95(26)$ & 0.003 \\
\hline Symptom onset time & & & & 0.426 \\
\hline$\leq 6$ hours & $98(22.8)$ & $147(26.3)$ & $26(24.5)$ & \\
\hline 6 hours -2 days & $87(20.3)$ & $123(22.0)$ & $27(25.5)$ & \\
\hline$>2$ days & $244(56.9)$ & $288(51.6)$ & $53(50.0)$ & \\
\hline ECG rhythm (\%) & & & & 0.021 \\
\hline Sinus & $219(51.2)$ & $316(56.6)$ & $70(66.7)$ & \\
\hline AF & $173(40.4)$ & $189(33.9)$ & $31(29.5)$ & \\
\hline Others & $36(8.4)$ & $53(9.5)$ & $4(3.8)$ & \\
\hline
\end{tabular}

LVEF at ED (\%)

0.294

$<35 \%$

$35-50 \%$

$>50 \%$

Physical examination (\%)

JVD

Orthopnoea

Rale

Peripheral oedema

Pulmonary oedema

Comorbidities (\%)

History of Heart Failure
Below

158 (39.8)

$113(28.5)$

$126(31.7)$

249 (59.3)

$260(60.9)$

298 (69.8)

$308(71.8)$

$317(73.9)$

269 (62.7)
$353(64.3) \quad 74(71.2) \quad 0.054$

$384(68.9) \quad 76(71.7) \quad 0.013$

$398(71.3)$

81 (76.4)

0.401

398 (71.5)

84 (79.2)

0.246

445 (79.7)

87 (82.1)

0.047
34 (35.1)

32 (33.0) 


\begin{tabular}{|c|c|c|c|c|}
\hline Hypertension & $286(66.7)$ & $388(69.5)$ & $71(67.0)$ & 0.608 \\
\hline Diabetes mellitus & $161(37.5)$ & $193(34.6)$ & $45(42.5)$ & 0.260 \\
\hline COPD & $41(9.6)$ & $60(10.8)$ & $8(7.5)$ & 0.561 \\
\hline Coronary artery disease & $144(33.6)$ & $162(29.0)$ & $33(31.1)$ & 0.312 \\
\hline \multicolumn{5}{|l|}{ Medication at admission (\%) } \\
\hline Loop diuretics & $288(67.1)$ & $183(32.8)$ & $65(61.3)$ & $<0.001$ \\
\hline $\begin{array}{l}\text { Loop diuretics dose among takers } \\
\text { (mg) }\end{array}$ & $40[40-60]$ & 20 [20-20] & $10[10-20]$ & $<0.001$ \\
\hline ACE-I & $79(18.4)$ & $81(14.5)$ & $22(20.8)$ & 0.130 \\
\hline ARB & $143(33.3)$ & $169(30.3)$ & $32(30.2)$ & 0.567 \\
\hline Beta blocker & $216(50.9)$ & $195(35.0)$ & $38(35.8)$ & $<0.001$ \\
\hline Aldosterone blocker & $117(27.3)$ & $80(14.3)$ & $20(18.9)$ & $<0.001$ \\
\hline \multicolumn{5}{|l|}{ Laboratory data at admission } \\
\hline White blood cell $(/ \mu \mathrm{L})$ & $\begin{array}{l}7,200[5,500- \\
9,900]\end{array}$ & $\begin{array}{l}8,000[6,000- \\
10,400]\end{array}$ & $\begin{array}{l}8,100[5,925- \\
10,675]\end{array}$ & 0.008 \\
\hline Albumin (g/dL) & $3.47(0.57)$ & $3.46(0.52)$ & $3.42(0.49)$ & 0.716 \\
\hline Haemoglobin (g/dL) & $11.6(2.26)$ & $12.0(2.34)$ & $11.7(2.08)$ & 0.007 \\
\hline AST (IU/L) & 33 [22-45] & $31[23-46]$ & $30[24-58]$ & 0.806 \\
\hline ALT (IU/L) & $21[14-33]$ & $22[14-36]$ & $20.50[13-36]$ & 0.557 \\
\hline Creatinine (mg/dL) & $\begin{array}{l}1.20[0.87- \\
1.64]\end{array}$ & $\begin{array}{l}1.02[0.78- \\
1.44]\end{array}$ & $\begin{array}{l}1.15[0.81- \\
1.92]\end{array}$ & $<0.001$ \\
\hline $\mathrm{BUN}(\mathrm{mg} / \mathrm{dL})$ & $26[19-37]$ & $23[17-31]$ & $25[19-35]$ & $<0.001$ \\
\hline Sodium (mEq/L) & 139 [137-142] & 140 [137-142] & 140 [137-142] & 0.422 \\
\hline Potassium (mEq/L) & $4.21(0.63)$ & $4.28(0.71)$ & $4.50(0.81)$ & 0.001 \\
\hline Glucose (mg/dL) & $163(76)$ & $169(76)$ & $184(90)$ & 0.049 \\
\hline $\mathrm{CRP}(\mathrm{mg} / \mathrm{dL})$ & $\begin{array}{l}0.56[0.20- \\
2.01]\end{array}$ & $\begin{array}{l}0.77[0.21- \\
2.26]\end{array}$ & $\begin{array}{l}0.84[0.32- \\
2.57]\end{array}$ & 0.115 \\
\hline $\mathrm{BNP}(\mathrm{pg} / \mathrm{mL})$ & $\begin{array}{l}757[439- \\
1,510]\end{array}$ & $\begin{array}{l}707[437- \\
1,254]\end{array}$ & $\begin{array}{l}827[409- \\
1,572]\end{array}$ & 0.373 \\
\hline $\begin{array}{l}\text { Total furosemide used within six } \\
\text { hours (mg) }\end{array}$ & $20[10-30]$ & $20[20-37]$ & $40[23-50]$ & $<0.001$ \\
\hline Urine output within $6 \mathrm{~h}(\mathrm{~mL})$ & $\begin{array}{l}755[465- \\
1168]\end{array}$ & $\begin{array}{l}900[580- \\
1,440]\end{array}$ & $\begin{array}{l}980[480- \\
1,370]\end{array}$ & $<0.001$ \\
\hline
\end{tabular}


Continuous variables are expressed as mean (standard deviation) or median [interquartile range].

ACE-I, angiotensin-converting enzyme inhibitor; AF, atrial fibrillation; ALT, alanine aminotransferase; $A R B$, angiotensin II receptor antagonist; AST, aspartate aminotransferase; BNP, brain natriuretic peptide; BUN, blood urea nitrogen; COPD, chronic obstructive pulmonary disease; CRP, C-reactive protein; ECG, electrocardiogram; ED, emergency department; JVD, jugular vein distention; LVEF, left ventricular ejection fraction

The total amount of IV furosemide used within the first six hours of admission differed significantly between the Below (20 mg; IQR, 10-30 mg), Standard (20 mg; IQR, 20-37 mg), and Above (40; IQR, 23$50 \mathrm{mg})$ groups $(P<0.001)$. Urine output measured during the first six hours of admission and DR are shown in Figure 1. The urine output within during the six hours of admission was significantly higher and DR significantly lower in the Above group ( $P<0.001$ for both). Univariate linear regression analysis showed that the DR in the Above group was significantly lower and in the Below group significantly higher than in the Standard group (Table 2). After adjusting for covariates shown to be related to DR within six hours, the DR in the Below group remained significantly higher than the Standard group, whereas the Above and Standard groups were no longer statistically different.

Table 2

Association between dose groups and diuretic response

\begin{tabular}{lllllll}
\hline Groups & \multicolumn{2}{l}{ Unadjusted model } & \multicolumn{3}{l}{ Adjusted model* } \\
\cline { 2 - 7 } & $\begin{array}{l}\text { Beta } \\
\text { coefficient }\end{array}$ & $95 \% \mathrm{Cl}$ & $\begin{array}{l}P \text { - } \\
\text { value }\end{array}$ & $\begin{array}{l}\text { Beta } \\
\text { coefficient }\end{array}$ & $95 \% \mathrm{Cl}$ & $\begin{array}{l}P_{\text {- }} \\
\text { value }\end{array}$ \\
\hline Standard & Reference & & & Reference & \\
\hline Below & 358.6 & $127.4-589.6$ & 0.002 & 371.1 & $\begin{array}{l}109.5- \\
632.8\end{array}$ & 0.006 \\
\hline Above & -612.5 & $-997.5--$ & 0.002 & -296.6 & $-686.2-$ & 0.135 \\
& & 227.5 & & & 92.9 & \\
\hline
\end{tabular}

* Adjusted for age, whether were taking oral loop diuretics before admission, white blood cell count, and levels of serum albumin, creatinine, potassium, and brain natriuretic peptide.

$\mathrm{Cl}$, confidence interval.

The median length of HS differed significantly between the Below (18 days; IQR, 11-26 days), Standard (15 days; IQR, 10-23 days), and Above (12 days; IQR, 7-22 days) groups ( $P<0.001$; Figure 2). Multiple linear regression analysis showed that the Below group had a significantly longer HS than in the Standard group (Table 3), while HS in the Above group was marginally but insignificantly shorter than in the Standard group. 


\section{Table 3}

Association between the dose groups and the length of hospital stay

\begin{tabular}{lllllll} 
Groups & \multicolumn{2}{l}{ Unadjusted model } & \multicolumn{3}{l}{ Adjusted model* } \\
\cline { 2 - 7 } & $\begin{array}{l}\text { Beta } \\
\text { coefficient }\end{array}$ & $95 \% \mathrm{Cl}$ & $\begin{array}{l}P \text { - } \\
\text { value }\end{array}$ & $\begin{array}{l}\text { Beta } \\
\text { coefficient }\end{array}$ & $95 \% \mathrm{Cl}$ & $P$-value \\
Standard & Reference & & & Reference & \\
\hline Below & 2.30 & $0.15-4.46$ & 0.036 & 2.38 & $0.05-4.70$ & 0.045 \\
\hline Above & -1.63 & $-5.26-$ & 0.376 & -3.34 & $-7.07-$ & 0.078 \\
& & 1.20 & & & 0.38 & \\
\hline
\end{tabular}

Those who died during the index hospitalisation were excluded.

* Adjusted for age, sex, history of heart failure, the New York Heart Association class, systolic blood pressure, haemoglobin, serum creatinine, sodium, albumin, and log-transformed BNP.

BNP, brain natriuretic peptide; $\mathrm{Cl}$, confidence interval.

Seventy-four deaths were observed during the 60 days of admission. Kaplan-Meier curves showed that the Above group was significantly associated with a higher 60-day mortality rate (Figure 3 ). The Cox regression analysis showed that the Above, but not the Below, group was associated with a significantly higher 60-day mortality rate than the Standard group in unadjusted and adjusted models (Table 4). As we have already shown that door-to-furosemide time was associated with 30-day mortality, ${ }^{6}$ we adjusted for the door-to-furosemide time in the multivariable Cox regression analysis, but results remained unchanged (Table 4). 


\section{Table 4}

Cox proportional hazard analysis for 60-day mortality according to the initial furosemide dose groups

$\begin{array}{lll}\text { Unadjusted } & \text { Adjusted for OPTIME- } & \begin{array}{l}\text { Adjusted for OPTIME-CHF } \\ \text { CHF Score + }\end{array} \\ \begin{array}{ll}\text { Log BNP } & \text { time }\end{array}\end{array}$

$\begin{array}{lllllllll}\mathrm{HR} & \begin{array}{ll}95 \% \\ \mathrm{Cl}\end{array} & \begin{array}{l}P- \\ \text { value }\end{array} & \mathrm{HR} & \begin{array}{l}95 \% \\ \mathrm{Cl}\end{array} & \begin{array}{l}P \text { - } \\ \text { value }\end{array} & \mathrm{HR} & 95 \% \mathrm{Cl} & \begin{array}{l}P \text { - } \\ \text { value }\end{array}\end{array}$

Dose group

\begin{tabular}{|c|c|c|c|c|c|c|c|c|c|}
\hline \multirow{2}{*}{$\begin{array}{l}\text { Standard } \\
\text { Below }\end{array}$} & \multicolumn{3}{|c|}{1 (Reference) } & \multicolumn{3}{|c|}{1 (Reference) } & \multicolumn{3}{|c|}{1 (Reference) } \\
\hline & 0.95 & $\begin{array}{l}0.57- \\
1.58\end{array}$ & 0.842 & 1.01 & $\begin{array}{l}0.48- \\
2.13\end{array}$ & 0.980 & 1.02 & $\begin{array}{l}0.48- \\
2.15\end{array}$ & 0.964 \\
\hline Above & 2.05 & $\begin{array}{l}1.09- \\
3.88\end{array}$ & 0.027 & 3.89 & $\begin{array}{l}1.70- \\
8.88\end{array}$ & 0.001 & 3.90 & $\begin{array}{l}1.71- \\
8.91\end{array}$ & 0.001 \\
\hline $\begin{array}{l}\text { OPTIME Score } \\
\text { (per } 1 \text { point) }\end{array}$ & & & & 1.01 & $\begin{array}{l}1.01- \\
1.02\end{array}$ & $<0.001$ & 1.01 & $\begin{array}{l}1.01- \\
1.02\end{array}$ & $<0.001$ \\
\hline D2F time & & & & & & & 0.99 & $\begin{array}{l}0.99- \\
1.01\end{array}$ & 0.739 \\
\hline
\end{tabular}

$\mathrm{BNP}$, brain natriuretic peptide; $\mathrm{Cl}$, confidence interval; $\mathrm{D} 2 \mathrm{~F}$, door to furosemide; $\mathrm{HR}$, hazard ratio; OPTIME, Outcomes of a Prospective Trial of Intravenous Milrinone for Exacerbations.

\section{Discussion}

This study demonstrated that the Standard group, receiving the guideline-recommended initial diuretic IV dose, was associated with a shorter HS than in the Below group, and a higher 60-day survival rate than in the Above group, even after adjusting for various confounders. To the best of our knowledge, this is the first study to validate the guideline-recommended initial IV loop diuretic dose in an AHF cohort.

DR was recently suggested as a metric of diuretic efficiency, and poor DR was reported to be an independent predictor of worse outcomes in patients with AHF. ${ }^{7,8}$ It is well known that dose-response curves of loop diuretics have a ceiling effect, suggesting that increasing the doses above a certain point will not increase the diuretic effect. ${ }^{9}$ This effect was clearly shown in our study. Our results showed that higher-than-suggested furosemide doses were associated with a significantly poorer DR. The use of a higher diuretic dose is a trade-off between achieving urine output and the subsequent decongestion and risking the downsides of diuretic use. Doses above the ceiling level could be more harmful than beneficial. On the other hand, doses below the diuretic dose recommended by guidelines had significantly greater DR; however, smaller doses were not associated with a better 60-day prognosis than the standard dose and were associated with a longer HS. This finding might suggest that lowering the diuretic dose will not directly lead to a better prognosis, even with a greater DR. Rather, it would result in longer HS, possibly because of the lower urine output achieved. Indeed, the HS after AHF in Japan was reported to 
be longer than in Western countries. One of the reasons for this difference could be that the diuretic doses given to patients with AHF in Japan are lower than in Western countries. ${ }^{10,11}$ As it happens, the guidelinerecommended furosemide dose is the suitable one, neither too high nor too low.

The DOSE trial, a prospective, double-blind, randomised controlled trial focusing on the usage of IV furosemide in patients with AHF, demonstrated conflicting results. That study found no statistical differences between using low and high doses in terms of 60-day composite mortality and rates of rehospitalisation and emergency visits for AHF. ${ }^{5}$ Although the current recommendations on the initial IV diuretic dose were primarily derived from the DOSE study, we could not simply compare the DOSE and our studies because they differ in some crucial elements. First, the DOSE trial enrolled patients with a history of chronic heart failure that took oral loop diuretics equivalent to 80 to $240 \mathrm{mg}$ furosemide, while the present study enrolled consecutive patients with AHF irrespective of whether the presented a de novo disease or were with chronic heart failure, and we did not limit the oral furosemide dose. Given that around half of the patients with AHF are with a newly diagnosed disease, our study seemed to better represent the real-world AHF population, making our results more clinically applicable. Second, the time from hospital arrival to IV furosemide use was quite different as well. We only enrolled patients who received the initial IV furosemide bolus within six hours of admission, whereas the patients in the DOSE trial were enrolled after a median time of 14.6 hours from arrival. Besides, most patients in the DOSE trial received the initial IV diuretics after arrival and before enrolment. Third, the IV furosemide dose regimen was different. A bolus infusion of furosemide was administered every 12 hours in the DOSE trial, and the daily dose was regarded as equal or high dose. This meant that a single IV furosemide dose in the DOSE trial was half of the daily equal or high dose. These facts indicated that the DOSE trial was inconclusive about the initial IV furosemide dose during the very acute phase of AHF.

\section{Strengths and limitations}

The strength of our study is that we evaluated the dose of diuretics standardised by the amount of diuretic prescribed before admission. Previous studies that simply evaluated the diuretic dose prescribed during hospitalisation and the prognosis could be heavily confounded by the disease severity of the patients, i.e., those treated with a higher dose of diuretics were older, with a history of heart failure, and prescribed higher doses. This bias could not be fully adjusted, even if a multivariable model was applied. We found no such association in our study between the Above group and a high-risk profile.

There are also several limitations that should be acknowledged. This study was not predefined. It was a post hoc analysis of registry data; therefore, the results should be interpreted cautiously. Additionally, a significant number of patients were excluded for a late administration of the first IV furosemide because we focused on the very early phase of AHF treatment. Furthermore, the groups differed in some baseline characteristics. The worse outcome of the higher dose might reflect disease severity rather than the impact of the initial IV furosemide dose. The results consistently showed an association between the initial IV furosemide dose and the outcome, even after adjusting for the OPTIME-CHF score and 
catecholamine use to minimise the disease severity effect. However, there could still be other unmeasured confounders.

\section{Conclusions}

Treating patients with AHF with guideline-recommended initial IV furosemide dose was associated with shorter hospital stay than with lower doses and a higher 60-day survival rate than with higher doses. Our study results endorse the current guidelines concerning the first IV furosemide dose in terms of prognosis and diuretic efficiency.

\section{Methods}

\section{Study design and patients}

The present study utilised data from the REALITY-AHF, a prospective multicentre registry focused on the presentation and treatment during the very early phase of AHF hospitalisation. Details on the study design were published elsewhere. ${ }^{6}$ Briefly, consecutive patients with AHF aged $\geq 20$ years hospitalised through the emergency department (ED) in 20 hospitals in Japan were enrolled. The AHF diagnosis was determined by an attending physician at each site, using the Framingham criteria. ${ }^{12}$ Patients with brain natriuretic peptide (BNP) $<100 \mathrm{ng} / \mathrm{L}$ or N-terminal pro b-type natriuretic peptide (NT-proBNP) $<300 \mathrm{ng} / \mathrm{L}$ were excluded due to diagnostic uncertainty, following the guidelines. ${ }^{3}$ Detailed inclusion and exclusion criteria and other study information are publicly available at the University Hospital Information Network (UMIN-CTR, unique identifier: UMIN000014105). Informed consent was obtained from all participants. The study protocol complied with the Declaration of Helsinki. It was first approved by the Kameda Medical Center, Clinical Research Committee (Kameda Medical Center, Research ethics committee), and subsequently approved by the ethical committee in each participating hospital before commencing patient enrolment (Tokyo Medical and Dental University, Research ethics committee; Nagoya University Graduate School of Medicine, Research ethics committee; St. Marianna University School of Medicine, Research ethics committee; Himeji Cardiovascular Center, Research ethics committee; Yokohama City University Medical Center, Research ethics committee; Fukushima Medical University, Research ethics committee; University of Tsukuba, Research ethics committee; the Sakakibara Heart Institute of Okayama, Research ethics committee; National Cerebral and Cardiovascular Center, Research ethics committee).

We analysed only patients treated with an IV bolus of furosemide within six hours of ED admission. Those treated with continuous furosemide infusion were excluded. We also excluded patients with hypotension (systolic blood pressure $<90 \mathrm{mmHg}$ ) at the time of ED admission. To validate the guidelinerecommended initial IV furosemide dose, we divided the cohort into three dose groups (Below, Standard, and Above) according to whether the initial IV furosemide dose was lower, equal to, or higher than the guideline-recommended dose of $40 \mathrm{mg}$ IV furosemide for patients with AHF not taking diuretics, or IV furosemide at the same dose as the oral loop diuretics for those already taking them. ${ }^{2,3}$ Doses of other 
oral loop diuretics that were considered equivalent to $20 \mathrm{mg}$ oral furosemide included $5 \mathrm{mg}$ torasemide and $30 \mathrm{mg}$ azosemide.

The primary endpoint was all-cause 60-day mortality. The evaluated secondary endpoints included diuretic response (DR) and length of hospital stay (HS). Baseline data, including physical findings, echocardiography, and laboratory tests, were collected at the ED. A DR was defined as urine output ( $\mathrm{mL}$ ) obtained during the first six hours per $40 \mathrm{mg}$ of IV furosemide (or equivalent).

\section{Statistical analysis}

Data are presented as mean \pm standard deviation or median and interquartile range (IQR) for continuous variables and as frequency (\%) for categorical variables. One-way analysis of variance or the KruskalWallis test was used to compare continuous variables. The $\chi^{2}$ or Fisher's exact test was used to compare categorical variables. When necessary, variables were transformed for further analyses.

We performed univariate and multivariable linear regression analyses to evaluate the association between the first furosemide IV dose and DR and the length of HS. The multivariable model for DR was adjusted for age, whether the patient took oral loop diuretics before admission, white blood cell count, and serum levels of albumin, creatinine, and potassium, having already shown them to be independently associated with DR in this cohort. ${ }^{8}$ The multivariable model for length of HS was adjusted according to the literature for age, sex, history of heart failure, the New York Heart Association (NYHA) class, systolic blood pressure, haemoglobin, serum levels of creatinine, sodium, and albumin, and log-transformed BNP.

Event-free survival curves were constructed using the Kaplan-Meier survival method and compared using log-rank statistics. The Outcomes of a Prospective Trial of Intravenous Milrinone for Exacerbations of Chronic Heart Failure (OPTIME-CHF) score was calculated for each patient as previously described ${ }^{13}$ to determine if the first furosemide IV dose was independently associated with mortality. The OPTIME-CHF risk score is based on age, the NYHA class, systolic blood pressure, and the levels of blood urea nitrogen and serum sodium. We used this score as an adjustment variable in the multivariable Cox model.

Moreover, recent studies showed that BNP level at admission was associated with the prognosis. ${ }^{14}$ Therefore, we also included the BNP level at admission as an adjustment variable.

All statistical analyses were performed using the R (The R Foundation for Statistical Computing, Vienna, Austria). In all analyses, a two-tailed $P$-value $<0.05$ indicated statistical significance.

\section{Declarations}

\section{Data availability}

The data underlying this article will be shared on reasonable request to the corresponding author. 


\section{Acknowledgments:}

None.

\section{Author contributions:}

K.Y. and Y.M. contributed to the conception or design of the work. T.O., K.K., S.O., E.A., S.S., M.Y., A.M., S.K., N.K., T.Y., T.S., A.M., T.K. and Y.M. contributed to the acquisition, analysis, or interpretation of data for the work. K.Y., D.M. and Y.M. drafted and critically revised the manuscript. All gave final approval and agree to be accountable for all aspects of work ensuring integrity and accuracy.

\section{Competing interests:}

Dr Takahoro Okumura received honoraria from Ono Yakuhin, Novartis, AstraZeneca, and Otsuka, and research grants from Ono Yakuhin, Amgen Astellas, Pfizer, Alexion, and Alnylam (not in connection with the submitted work). Dr Keisuke Kida received honoraria from Daichi Sankyo Co., Ono Yakuhin, AstraZeneca, Otsukaand Novartis. Dr. Nobuyuki Kagiyama is affiliated with a department funded by Philips Healthcare, Asahi KASEI Corporation, Inter Reha Co., Ltd, and Toho Holdings Co., Ltd based on collaborative research agreement. Dr. Yuya Matsue is affiliated with a department endowed by Philips Respironics, ResMed, Teijin Home Healthcare, and Fukuda Denshi. Dr. Yuya Matsue received honorariums from Otsuka Pharmaceutical Co. and Novartis Japan. The remaining authors have no conflicts of interest to declare.

\section{Funding:}

REALITY-AHF was funded by The Cardiovascular Research Fund, Tokyo, Japan. The funding sources had no role in the design and conduct of the study; collection, management, analysis, and interpretation of the data; preparation, review, or approval of the manuscript; and decision to submit the manuscript for publication.

\section{References}

1. Mentz, R. J. et al. Decongestion in acute heart failure. Eur J Heart Fail, 16, 471-482 (2014).

2. Yancy, C. W. et al. 2013 ACCF/AHA guideline for the management of heart failure: executive summary: a report of the American College of Cardiology Foundation/American Heart Association Task Force on practice guidelines. Circulation 128, 1810-1852 (2013).

3. Ponikowski, P. et al. 2016 ESC Guidelines for the diagnosis and treatment of acute and chronic heart failure: The Task Force for the diagnosis and treatment of acute and chronic heart failure of the 
European Society of Cardiology (ESC). Developed with the special contribution of the Heart Failure Association (HFA) of the ESC. Eur J Heart Fail 18, 891-975 (2016).

4. Felker, G. M., O'Connor, C. M. \& Braunwald, E. \& Heart Failure Clinical Research Network, I. Loop diuretics in acute decompensated heart failure: necessary? Evil? A necessary evil? Circ Heart Fail, 2, 56-62 (2009).

5. Felker, G. M. et al. Diuretic strategies in patients with acute decompensated heart failure. N Engl J Med, 364, 797-805 (2011).

6. Matsue, Y. et al. Time-to-Furosemide Treatment and Mortality in Patients Hospitalized With Acute Heart Failure. J Am Coll Cardiol, 69, 3042-3051 (2017).

7. Voors, A. A. et al. Diuretic response in patients with acute decompensated heart failure: characteristics and clinical outcome-an analysis from RELAX-AHF. Eur J Heart Fail, 16, 1230-1240 (2014).

8. Kuroda, S. et al. Very Early Diuretic Response After Admission for Acute Heart Failure. J Card Fail, 25, 12-19 (2019).

9. Oh, S. W. \& Han, S. Y. Loop Diuretics in Clinical Practice. Electrolyte Blood Press, 13, 17-21 (2015).

10. Konishi, M. et al. Heart failure epidemiology and novel treatments in Japan: facts and numbers. ESC Heart Fail, 3, 145-151 (2016).

11. Tanaka, T. D., Sawano, M., Ramani, R., Friedman, M. \& Kohsaka, S. Acute heart failure management in the USA and Japan: overview of practice patterns and review of evidence. ESC Heart Fail, 5, 931947 (2018).

12. Ho, K. K., Anderson, K. M., Kannel, W. B. \& Grossman, W. \& Levy, D. Survival after the onset of congestive heart failure in Framingham Heart Study subjects., 88, 107-115 (1993).

13. Felker, G. M. et al. Risk stratification after hospitalization for decompensated heart failure. $J$ Card Fail, 10, 460-466 (2004).

14. Santaguida, P. L. et al. BNP and NT-proBNP as prognostic markers in persons with acute decompensated heart failure: a systematic review. Heart Fail Rev, 19, 453-470 (2014).

\section{Figures}



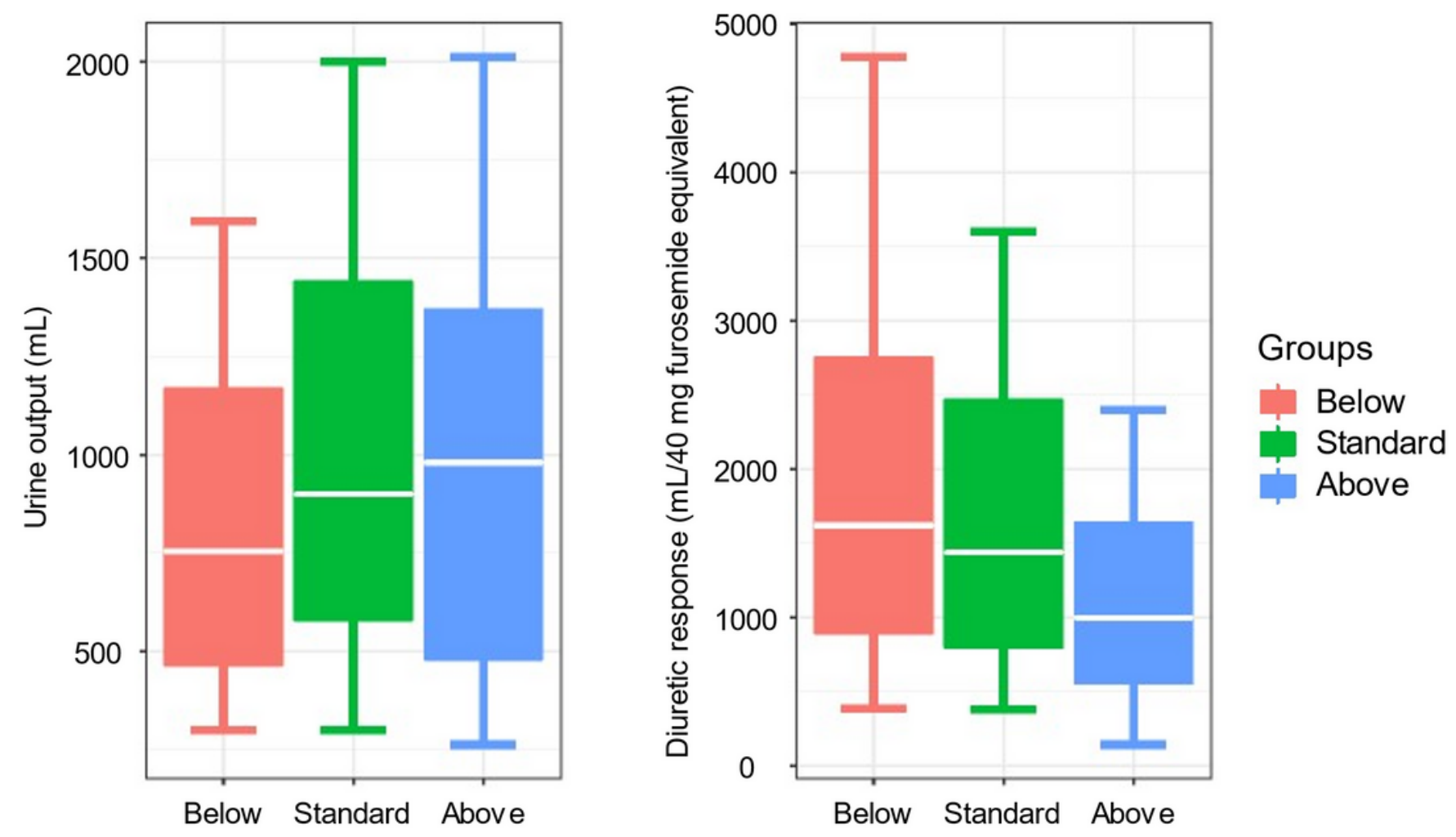

\section{Figure 1}

Urine output during the first six hours and diuretic response Diuretic response was defined as the urine volume during the first six hours per $40 \mathrm{mg}$ of IV furosemide. Although the urine output during the first six hours in the Above group was significantly larger than in the Standard group, its diuretic response was significantly lower $(P<0.001$ for both). 


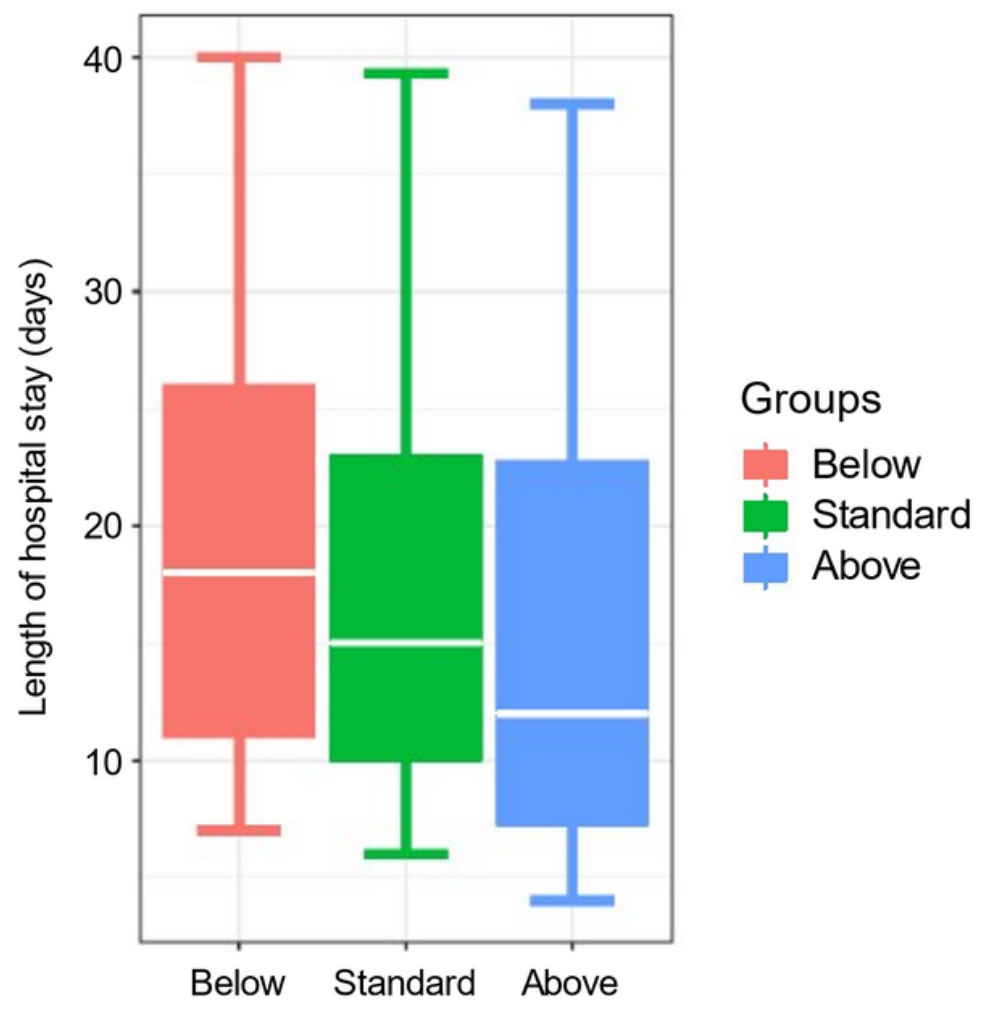

Figure 2

Length of hospital stay according to the first furosemide IV dose The hospital stay in the Below group was significantly longer than in the other groups $(P<0.001)$. 


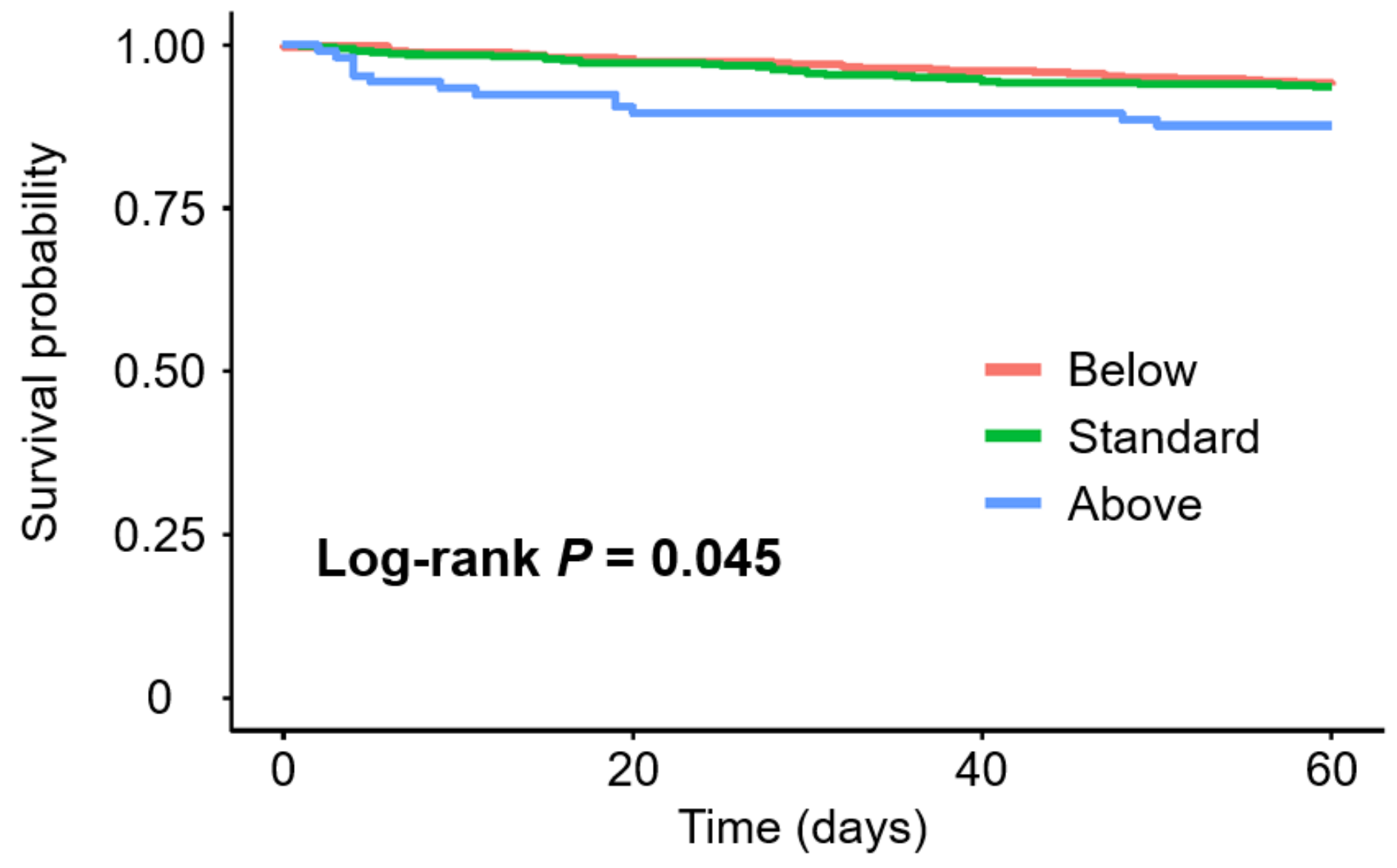

Number at risk

Below 429

Standard 558

Above 106
419

534

95
406

514

93
396

505

91

Figure 3

Kaplan-Meier curves for 60-day mortality according to the first furosemide IV dose The Above group was significantly associated with a lower survival rate.

\section{Supplementary Files}

This is a list of supplementary files associated with this preprint. Click to download.

- SuupplementalFigureS1SR.docx 